\title{
Successful Endoscopic Reduction of an Ileocolonic Intussusception in an Adult With Peutz-Jeghers Syndrome
}

\author{
Mohd Amer AlSamman ${ }^{a, d}$, Jason D. Ferreira ${ }^{b}$, Abdelmoniem Moustafa $^{a}$, \\ Jonathan Moveson ${ }^{\mathrm{c}}$, Paul Akerman ${ }^{\mathrm{b}}$
}

\begin{abstract}
Peutz-Jeghers syndrome (PJS) is a rare autosomal dominant disorder characterized by hamartomatous polyps throughout the gastrointestinal tract, mostly causing gastrointestinal bleeding and recurrent intestinal obstructions. Few intussusception related to PJS occurs reportedly in adults. Unlike pediatric cases almost all reported cases end up with surgical resection. Here we present a case of PSJ-related intussusception in an adult patient treated successfully with endoscopic approach, with no surgical intervention required.
\end{abstract}

Keywords: Intussusception; Peutz-Jeghers syndrome; Endoscopy

\section{Introduction}

Peutz-Jeghers syndrome (PJS) is a rare autosomal dominant disorder characterized by hamartomatous polyps throughout the gastrointestinal tract, mostly confined to the small bowel (60-90\%) and the colon (50-64\%) [1]. These polyps have been reported to cause gastrointestinal bleeding, can lead to iron deficiency anemia in $14 \%$ of patients and cause recurrent intestinal obstructions in $43 \%$ of patients [1]. Most reported cases of intussusception related to PJS occur in children with only $5 \%$ reportedly occurring in adults. Overall, PJS complications contribute to only $1 \%$ of all causes of intestinal obstruction [2] and $0.003-0.02 \%$ of all hospital admissions.

Given the chronic, intermittent and nonspecific gastrointestinal symptoms associated with PJS, diagnosis can be delayed and sometimes cases are first diagnosed at emergency

Manuscript submitted December 15, 2018, accepted January 21, 2019

aThe Warren Alpert Medical School of Brown University, The Miriam Hospital, Providence, RI, USA

bivision of Gastroenterology, The Warren Alpert Medical School of Brown University, Providence, RI, USA

${ }^{\mathrm{c}}$ The Warren Alpert Medical School of Brown University, Radiology Department, The Miriam Hospital, Providence, RI, USA

${ }^{\mathrm{d} C}$ Corresponding Author: Mohd Amer AlSamman, The Warren Alpert Medical School of Brown University, The Miriam Hospital, Providence, RI 02906, USA. Email: alsamman.amer@gmail.com

doi: https://doi.org/10.14740/gr1130 laparotomy for bowel obstruction. With more frequent use of computed tomography (CT) in the evaluation of patients with abdominal pain, the condition can be diagnosed more readily. Treatment entails simple bowel resection in most cases and the role of endoscopic reduction of the intussusception remains controversial [3]. Endoscopic treatment for PJS-related intussusception has been described in a few pediatric cases, but to our knowledge this intervention has not been previously described in adults. Here we present a case with successful endoscopic reduction of ileocolic intussusception with no complications or recurrences after 1 year of followup.

\section{Case Report}

A 19-year-old male patient presented with known PJS with no previous abdominal surgeries and known history of multiple small intestinal and colonic polyps removed on previous endoscopies, and the last colonoscopy was 6 years ago. He presented with new onset right-sided abdominal pain, nausea, and bilious vomiting. Laboratory workup revealed a hemoglobin of $10 \mathrm{~g} / \mathrm{dL}$, hematocrit of 36 , and mean corpuscular volume (MCV) of $62 \mathrm{fL} /$ cell but was otherwise unremarkable including normal liver function tests and electrolytes. He then underwent a contrast enhanced CT scan of the abdomen and pelvis that showed colo-colonic intussusception with fecalized terminal ileum, and multiple thickened hyperenhancing loops of jejunum with a $2.7 \times 1.7 \mathrm{~cm}$ lobulated cecal polyp seen adjacent to the ileocecal valve (Fig. 1). After evaluation by the surgical service, it was decided that endoscopic management should be tried before performing laparotomy. He then underwent colonoscopy that showed a $35 \mathrm{~mm}$ pedunculated polyp in the transverse colon not noted on imaging as well as the aforementioned cecal polyp that were both endoscopically resected using a hot snare. Following polyp resection, approximately $20 \mathrm{~cm}$ of intussuscepted ileum was seen in the ascending colon which was then endoscopically reduced using an inflated biliary stone extraction balloon over a guidewire placed through the ileocecal valve to guide the intussuscepted ileum back to its natural position. The ileocecal valve and ileum were noted to be mildly edematous but no ischemic changes were noted (Fig. 2). Following the procedure, the patient's symptoms completely resolved and he required no further intervention. Pathology from the resected polyps confirmed that they were hamartomatous in nature fit- 


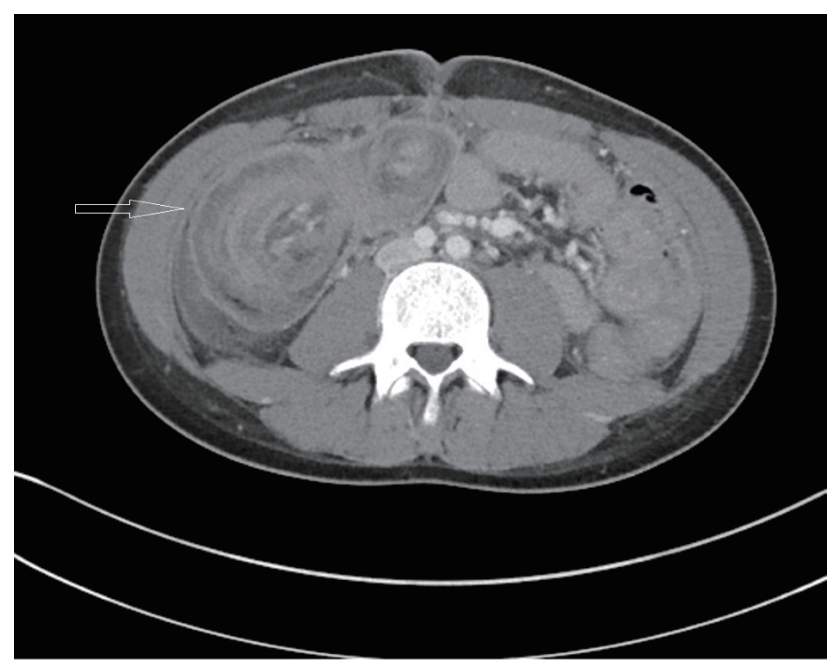

Figure 1. A contrast enhanced CT scan of the abdomen and pelvis showing colo-colonic intussusception with fecalized terminal ileum, and multiple thickened hyperenhancing loops of jejunum (target sign), and a $2.7 \times 1.7 \mathrm{~cm}$ lobulated cecal polyp seen adjacent to the ileocecal valve.

ting with his underlying PJS. Six months following hospital discharge, he underwent both upper and retrograde spiral enteroscopy that showed multiple hamartomatous polyps in the small bowel with selected resection of the larger sized polyps. Over a year has passed since his successful endoscopic intussusception reduction and he has remained free of complication or recurrence.

\section{Discussion}

Cases of adult intussusception are different from pediatric episodes in various aspects. In children, intussusception can usually be managed successfully with supportive care or pneumatic/hydrostatic (air contrast enema) reduction in $80 \%$ of patients. In contrast, almost $90 \%$ of intussusception cases in adults are secondary to a pathologic condition that serves as a lead point, such as carcinomas, polyps, Meckel's diverticulum, colonic diverticulum, strictures, or benign neoplasms that oftentimes require intervention. Although Aydin et al have reported successful treatment in selected adult cases without frank bowel obstruction with supportive care, the available data looking at conservative management for adult intussusception are limited because the etiology of the intussusception was not determined in these patients and there is a lack of long term follow-up to assess for recurrence $[4,5]$. Further study by Lvoff et al performed analysis of several intussusception cases treated with conservative versus surgical management and postulated that the length of intussuscepted bowel detected on $\mathrm{CT}$ is the main factor in distinguishing those cases that are self-limited and can be monitored conservatively (less than $3.5 \mathrm{~cm}$ of intussuscepted bowel) from those that will require surgery (greater than a $3.5-\mathrm{cm}$ segment of intussuscepted bowel) $[4,5]$. Nonetheless, it remains questionable whether $\mathrm{CT}$ alone can be relied upon to determine

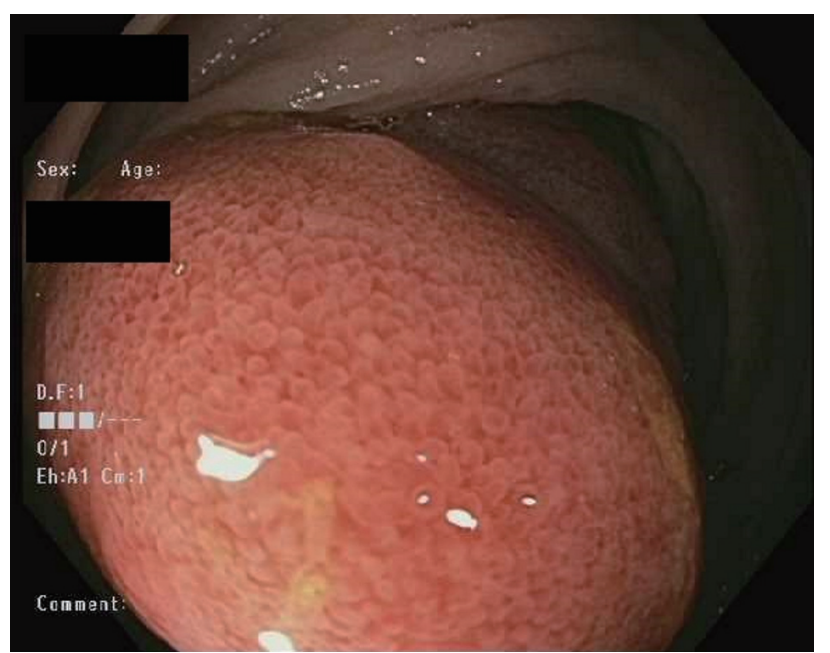

Figure 2. Endoscopic image showing that ileocecal valve and ileum were noted to be mildly edematous with no ischemic changes.

management of this condition as revealed in our case in which the imaging demonstrated colo-colonic intussusception when in fact the patient had ileo-colonic intussusception endoscopically.

Endoscopic reduction and radiologic decompression have traditionally not been the standard of care for adults and therefore $70-90 \%$ of cases are treated with definitive surgical resection or laparoscopic reduction [6]. Existing literature describes a high risk of perforation and recurrence after endoscopic intussusception reduction, which could possibly be due to inflammatory or ischemic changes in the bowel following intussusception $[7,8]$. Some literature describes a benefit of combined endoscopic and surgical laparotomy for polyps more than $15 \mathrm{~mm}$ in PJS patients [9]; however, this literature does not shed light on the incidence of long term complications of surgical treatment such as adhesions leading to future small bowel obstructions and incisional hernias.

In contrast to other reported cases, our patient had $20 \mathrm{~cm}$ of intussuscepted bowel and clinical features of bowel obstruction that was successfully treated with endoscopic management in the acute setting. Subsequent upper and retrograde spiral enteroscopies were then able to detect and resect all clinically significant polyps without complication or intussusception recurrence after over a year of clinical follow-up without the need for any surgical intervention. Factors that contributed to successful endoscopic management in this patient were rapid diagnosis and intervention prior to the bowel integrity becoming compromised by necrosis and the availability of spiral enteroscopy to remove further clinically significant polyps to try to prevent recurrence. Surgical management has been the gold standard of care for intussusception in adults, but in the era of more advanced and less invasive endoscopic treatments, more consideration should be made to move in this direction especially when patients present early prior to development of bowel compromise and in conditions such as PJS where the lead point of the intussusception is a hamartomatous polyp which is known to be amenable to endoscopic intervention. The development of newer endoscopic techniques allows in- 
terventional endoscopists to safely and minimally invasively manage traditional surgical conditions, and future studies in the modern era should analyze patient outcomes in comparison to a surgical approach.

\section{Financial Support}

None.

\section{Conflict of Interest}

None.

\section{References}

1. Beggs AD, Latchford AR, Vasen HF, Moslein G, Alonso A, Aretz S, Bertario L, et al. Peutz-Jeghers syndrome: a systematic review and recommendations for management. Gut. 2010;59(7):975-986.

2. Thakker HH, Joshi A, Deshpande A. Peutz-Jegher's syndrome presenting as jejunoileal intussusception in an adult male: a case report. Cases J. 2009;2:8865.
3. Yalamarthi S, Smith RC. Adult intussusception: case reports and review of literature. Postgrad Med J. 2005;81(953):174-177.

4. Aydin N, Roth A, Misra S. Surgical versus conservative management of adult intussusception: Case series and review. Int J Surg Case Rep. 2016;20:142-146.

5. Lvoff N, Breiman RS, Coakley FV, Lu Y, Warren RS. Distinguishing features of self-limiting adult smallbowel intussusception identified at CT. Radiology. 2003;227(1):68-72.

6. Marinis A, Yiallourou A, Samanides L, Dafnios N, Anastasopoulos G, Vassiliou I, Theodosopoulos T. Intussusception of the bowel in adults: a review. World J Gastroenterol. 2009;15(4):407-411.

7. Fazio RA, Wickremesinghe PC, Arsura EL, Rando J. Endoscopic removal of an intussuscepted appendix mimicking a polyp - an endoscopic hazard. Am J Gastroenterol. 1982;77(8):556-558.

8. Chang FY, Cheng JT, Lai KH. Colonoscopic diagnosis of ileocolic intussusception in an adult. A case report. S Afr Med J. 1990;77(6):313-314.

9. Kouklakis G, Kountouras J, Moschos J, et al. Combined endoscopic and surgical treatment for Peutz-Jeghers syndrome: a case report. Ann Gastroentol Hepatol. 2003;16(4):370-372. 